\title{
Phylogenetic Relationships of Epinephelus fuscoguttatus and Epinephelus hexagonatus Inferred from Mitochondrial Cytochrome b Gene Sequences using Bioinformatic Tools.
}

\author{
Baharum S. N.* and Nurdalila A. A.
}

\begin{abstract}
Molecular markers can be identified via a DNA test regardless of the developmental stage, age, or environmental challenges experienced by the organism. The degree of conservation of sequence using the cytochrome $b$ gene from mitochondrial DNA is sufficient to discriminate between closely related species. A partial DNA sequence of cytochrome b gene was used to investigate the molecular relationship between Epinephelus hexagonatus and Epinephelus fuscoguttatus. Polymerase Chain Reaction (PCR) and sequence analysis were used to determine the genetic variation in approximately 450-nucleotides region of the mitochondrial cytochrome b gene in 160 fresh tissue samples. Phylogenetic trees were constructed using bioinformatic tools with ME method, NJ method and MP method with bootstrapping using arithmetic average method. There are no differences or only small differences were detected between these two taxa. The results showed that Epinephelus fuscoguttatus clustered with Epinephelus hexagonatus, based on the phylogenetic trees Epinephelus hexagonatus and Epinephelus fuscoguttatus consistently clustered together with following the suggestion referring on their morphological data, that they may be closely related, or possibly placed within the same species.
\end{abstract}

Index Terms-Cytochrome b, Epinephelus fuscoguttatus, Epinephelus hexagonatus, phylogenetic tree.

\section{INTRODUCTION}

Groupers scientifically known as Epinephelus spp., have desirable taste with high market value and demands are one of the most important commercial of mariculture fish species in Asia and around the world [1]. They are fastgrowing, disease-resistant and suitable for culture, indicating that they possess excellent biological characteristics. Grouper farming appeared to have great promise, and coastal cage culture has potential for continued development. However, growth of the grouper culture industry has been hindered by an unreliable and limited supply of seed of the Epinephelus species. Controlled reproduction from simultaneously available mature broodstock of both sexes is required for the mass production of grouper fry in hatcheries, and this will certainly be the key to continued growth of the industry [2]. Across hundreds of millions years of evolution, major changes in regulatory genes have produced important evolutionary

Manuscript received April 11, 2011

Baharum S. N. and Nurdalila A. A. are with Institute of Systems Biology Universiti Kebangsaan Malaysia, UKM Bangi Selangor, Malaysia *Corresponding author: Baharum S. Nataqain. Email: nataqain@ukm.my changes in body plan and morphology, which is why the molecular marker approach has been essential for finding and tracking ancient and new species and especially for phylogenetic tree development.

Reference [3] studied of 28 grouper species within six genera of the subfamily Epinephelinae and used cytochrome $\mathrm{b}$ to derive the phylogenetic relationships of groupers in the China Seas. They concluded that the genus Plectropomus (Serranidae, Perciformes), which diverged early, is the most primitive group in the subfamily Epinephelinae; the genus Variola (serranidae, Perciformes) is more closely related to Cephalopholis (Serranidae, Perciformes) than to the other four genera; Cephalopholis is a monophyletic genus and more primitive than Epinephelus; Promicrops lanceolatus (Serranidae,Percoidei) and Cromileptes altivelis (Chromileptes, Serranidae) should be included in the genus Epinephelus; and there exist two sister groups in Epinephelus. The phylogenetic relationships among the fishes in the perciform tribe Epinephelinae (Epinephelus, Serranidae) have long been poorly understood in large part because of the numerous taxa that must be considered and the large, circumtropical distribution of the group. Knowledge of relationships within the Serranidae has been equally tenuous [4].

According to reference [5] molecular data have been proven to be very useful to clarify any taxonomic relationships. It is also can be used to define the species boundaries in morphologically conservative or highly variable groups. Mitochondrial DNA sequences also have proven to be effective for elucidating any phylogenetic relationship and taxonomic relationships. In addition, the growing popularity of sequence data to study the interspecific and intraspecific phylogeny requires the need for primer sets to target gene regions that appropriate for the taxonomic resolution at various levels has been increasing lately.

It has been shown that the cytochrome $b$ gene is accumulating the changes in nucleotide at a sufficient rate which can resolve the phylogenetic relationships among any closely related species [6]

Recently a few questions were raised on the Epinephelus species on their morphological similarities and the species had extensive phonetic similarities, suggesting that some species in Epinephelinae sp. might belong in a same species and group [7]. However, in a previous molecular pylogenetic study focusing on serranid fish genus Epinephelus [8] and Plectropomus [6] showed no or only provided general data on Epinehelus hexagonatus in their studies.

In our study, we carried out cytochrome b (cyt b) analysis 
as molecular marker to investigate the molecular relationship between Epinephelus hexagonatus and Epinephelus fuscoguttatus. Analysis from BLAST database of partial sequences of mtDNA cyt b of Epinephelus fuscoguttatus showed $99 \%$ similarity to the Epinephelus hexagonatus and that this two species are closely related and the level of differentiation on morphology between them is very low. This database has trigger a question, is these two species is a same species but have different name? Therefore, this study will carry out experiments that work on identifications and differentiations between those two species to answer some of the question that had arose time to time.

\section{MATERIALS AND METHODS}

\section{A. Collection and Preservation}

A piece of caudal fin area (20-50 mg) was individually collected from a live single Epinephelus fuscoguttatus. The specimens were collected from the Fisheries Research Institute, Terengganu Malaysia. A total of 160 individual initially identified as Epinephelus fuscoguttatus were subjected to the study. The specimens preserved in $95 \%$ ethanol at ambient temperature upon sampling and kept at $20^{\circ} \mathrm{C}$ under laboratory conditions. The specimens were homogenized in chilled nuclei lysis solution and kept at $20^{\circ} \mathrm{C}$ prior to be used.

\section{B. DNA Extraction}

DNA extraction was based on the commercial kit from Promega with some modification according to the tissues (blood, fin or liver). DNA quantity was determined against $1 \mathrm{~kb}$ molecular marker standard by electrophoresis in a $1 \%$ of agarose gel.

\section{PCR Amplification}

Polymerase Chain Reaction (PCR) was used to amplify approximately $490 \mathrm{bp}$ fragment of the mtDNA cyt b gene. In both genes double-stranded DNA was PCR synthesized in $25 \mu \mathrm{l}$ reactions $\left(5 \mu \mathrm{l} 5 \mathrm{X}\right.$ Green GoTaq ${ }^{\circledR}$ Flexi Buffer, $2 \mu \mathrm{l}$ $25 \mathrm{mM} \mathrm{MgCl}_{2}, 1 \mu \mathrm{l} 10 \mathrm{mM}$ of each primer, $0.5 \mu \mathrm{dNTP} 0.2$ $\mathrm{mM}$ /dinucleotide, $1 \mu \mathrm{l}$ template DNA, and $0.5 \mu \mathrm{l}(5 \mathrm{u})$ GoTaq ${ }^{\circledR}$ DNA Polymerase (Promega Corporation(C).

30 cycles of the following step (Table 1) procedure were performed using Eppendorf Master Cycler, the thermo cycler conditions were: preheat at $94{ }^{\circ} \mathrm{C}$ for $120 \mathrm{~s}$ (seconds), denaturation $94{ }^{\circ} \mathrm{C}$ for $5 \mathrm{~min}$, annealing $45^{\circ} \mathrm{C}$ for $2 \mathrm{~min}$, extension $72{ }^{\circ} \mathrm{C}$ for $1 \mathrm{~min}$, repeated for 1 cycle, followed by 29 cycles at $94{ }^{\circ} \mathrm{C}$ for $5 \mathrm{~min}, 45^{\circ} \mathrm{C}$ for $2 \mathrm{~min}, 72{ }^{\circ} \mathrm{C}$ for 1 min and hold in $4^{\circ} \mathrm{C}$. A set of primer used was: CytbFR 5'CGCCTGTTTATCAAAAACAT-3' and CytbRV 5'CCGGTCTGAACTCAGATCACGT-3’ [9].

\section{Sequencing}

PCR amplification products were purified on a $1 \%$ of agarose gel stained with Ethidium bromide (EtBr). Products of interest were identified using HindIII DNA ladder as a reference marker. PCR fragments were separated from the gel using a Wizard-prep PCR purification kit (Promega Corporation(C). Automated fluorescent dye-deoxy sequencing of both strands was carried out using an $\mathrm{ABI}$ Prism 3100 DNA-Sequencer. Sequences from both strands of all individuals from each species were assembled into a single consensus sequence using the assembly editor option in the Biology Workbench (ver. 3.2) and the tools that were used known as Nucleic Tools which combine general sequence analysis applications, with applications that are specifically for analyzing DNA or RNA and the second tool that were used known as Alignment Tools which allow to view and analyze aligned sequences.

\begin{tabular}{lll} 
TABLE 1. & $\begin{array}{c}\text { THERMAL CYCLING PROFILE USED TO AMPLIFY CYT B } \\
\text { GENE REGION AND PCR PRIMERS INVOLVED. }\end{array}$ \\
\hline Step & Temp. $\left({ }^{\circ} \mathbf{C}\right)$ & Time (s) \\
\hline Initial denaturation & 94 & 120 \\
Next 3 steps for 30 cycles & & 300 \\
Denaturation & 94 & 120 \\
Annealing & 45 & 60 \\
Extension & 72 & 60 \\
Final extension & 72 & \\
& & \\
PCR primers & & 5'-CGCCTGTTTATCAAAAACAT-3' \\
CytbFR & 5'-CCGGTCTGAACTCAGATCACGT-3' \\
CytbRV &
\end{tabular}

Consensuses of the sequences were aligned using the alignment program Clustal W with default settings [10]. Indels were coded as a single character so as not to place undue character weight on a single mutational event leading to the insertion or deletion of multiple bases and was excluded from the analysis due to the ambiguity of the aligned characters.

\section{E. Phylogenetic Analysis}

Epinephelus bruneus (accession No: NC013820) was used as an out-group when constructing the phylogenetic tree. Since variation within the nuclear loci was low, relationships between sequences for each locus were determine and the data set was analyzed with maximum parsimony (MP), molecular evolution (ME), and neighborjoining (NJ) using the program MEGA 4.0 [11]. Formulas for computing these quantities are as follows:

$d \quad$ : Transitions + Transversions : Proportion of nucleotide sites that are different.

$s \quad$ : Transitions only : Proportion of nucleotide sites with transitional differences.

$v \quad:$ Transversions only : Proportion of nucleotide sites with transversional differences.

$R=s / v \quad:$ Transition/transversions ratio.

$L \quad$ : No of valid common sites: Number of sites compared.

$p, n_{d} / L, p(1-p) / L$

$S, P, s(1-s) / L$

$V, Q, v(1-v) / L$

$R, P / Q,\left[C^{2}{ }_{1} P+C^{2}{ }_{2} Q-\left(C_{1} P+C_{2} Q\right)^{2}\right] / L$

where $C_{1}=1 / s$ and $C_{2}=-s / v^{2}$ 
$P$ and $Q$ are the proportion of sites showing transitional and transversional differences, respectively.

Sites with missing data were removed and the mitochondrial region sequences were used to test models of evolution. The p-distance model of evolution was used and employed to MP, ME and NJ tree the nucleotide sequences and relations were analyzed $\mathrm{MP}, \mathrm{ME}$ and $\mathrm{NJ}$ as implemented in MEGA 4.0 [11]. Support for nodes was estimated using the bootstrap technique with 100 replicates. All new sequences were deposited in Genbank as in Table 2.

TABLE 2. LIST OF TAXA SEQUENCED AND THEIR GENBANK ACCESSION NUMBERS

\begin{tabular}{lc}
\hline \multicolumn{1}{c}{ Label of Fish Samples } & GenBank Accession No. \\
\hline Epinephelus hexagonatus $3 C 11$ & GU591708 \\
Epinephelus hexagonatus $3 C 27$ & GU591711 \\
Epinephelus hexagonatus $4 C 7$ & GU591702 \\
Epinephelus hexagonatus $4 C 10$ & GU591703 \\
Epinephelus hexagonatus 4C13 & GU591704 \\
Epinephelus hexagonatus 4C17 & GU591705 \\
Epinephelus hexagonatus 5C18 & GU591718 \\
Epinephelus hexagonatus $6 C 6$ & GU591720 \\
\hline
\end{tabular}

\section{RESULTS}

The cytochrome $\mathrm{b}$ gene was partially sequenced for all individuals examined. The numbers of base substitutions per site from analysis between sequences are based on the pairwise analyses of 160 sequences. The evolutionary history was inferred using the Neighbour-Joining (NJ), Minimum Evolution (ME), and Maximum Parsimony (MP).

For the Maximum Parsimony (MP) method, this tree (Fig. 1) out of 2412 most parsimonious trees (length $=179$ ) is shown. The consistency index is (0.666667), the retention index is $(0.954545)$, and the composite index is 0.922550 (0.636364) for all sites and parsimony-informative sites (in parentheses). The MP tree was obtained using the Close-Neighbor-Interchange algorithm [13] with search level 3 [12]; [13] in which the initial trees were obtained with the random addition of sequences (100 replicates).

While the Minimum Evolution (ME) method, The ME tree (Fig. 2) was searched using the Close-NeighborInterchange (CNI) algorithm at a search level of 3. The evolutionary history was inferred using the Minimum Evolution method. The optimal tree with the sum of branch length $=0.51978324$ is shown. The percentage of replicate trees in which the associated taxa clustered together in the bootstrap test (100 replicates) is shown next to the branches. The phylogenetic tree was linearized assuming equal evolutionary rates in all lineages. The tree is drawn to scale, with branch lengths in the same units as those of the evolutionary distances used to infer the phylogenetic tree. All positions containing gaps and missing data were eliminated from the dataset (Complete deletion option). There were a total of 340 positions in the final dataset

Last but not least, the Neighbor-joining algorithm [14] was used to generate the initial tree. The evolutionary history that inferred using the Neighbor-Joining method showed the optimal tree (Fig. 3) with the sum of branch length $=0.95095399$. The bootstrap consensus for
Maximum Parsimony (MP), Minimum Evolution (ME) and Neighbor-Joining (NJ) tree inferred from 100 replicates is taken to represent the evolutionary history of the taxa analyzed [12].

These trees branches corresponding to partitions reproduced in less than $60 \%$ bootstrap replicates are collapsed and the percentage of replicate trees in which the associated taxa clustered together in the bootstrap test (100 replicates) is shown next to the branches [12]. All positions containing gaps and missing data were eliminated from the dataset (Complete deletion option).

There were a total of 340 positions in the final dataset for every set of the trees and out of which 8 were parsimony informative for the MP tree. These Phylogenetic analyses were conducted in MEGA4 [11].

\section{DISCUSSION}

Mitochondrial DNA is a crucial marker allowing researchers to recognize and identify this Serranidae species for the many advantages that it offers. For evaluating genetic diversity and phylogeny, modern molecular biology has enabled comparisons between nucleotide and amino acid sequences of different populations. Many studies such as [15] successfully studied on Osmeridae, reference [16] on Epinephelus quoyanus, reference [16] on yellow grouper, reference [6] on Epinephelus and Mycteroperca species, reference [18] on Cottidae, and reference [19] on Glyptosternoid species have demonstrated that polymorphic loci or gene sequences are often conserved between closely related taxa. As a genetic marker for phylogenetic analyses, mitochondrial DNA has many advantages.

The cytochrome b (cyt b) gene has been sequenced more often than any other mitochondrial gene [20]. Partial nucleotide sequences of cytochrome $b$ gene were aligned together and three types of phylogenetic trees were constructed using the Maximum Parsimony (MP), Molecular Evolution (ME) and Neighbour-Joining (NJ). The topologies of MP (Fig. 1), ME (Fig. 2) and NJ (Fig. 3) trees were similar. The 160 individual of samples were clustered into 3 cluster groups with high bootstrap and consensus resampling support. Those 3 groups can be occurred with so many possibilities; some of the possibilities are variations on the tandom repeats [21], mutations (deletion/insertion/substitution) on the genes [22], genetic drifts as a result of small effective population sizes ecological diversity [23] and many more. These possibilities can also be used to describe the interference of Epinephelus hexagonatus into the cluster of Epinephelus fuscoguttatus.

Based on the figure of the phylogenetic tree of MP (Fig. 1), ME (Fig. 2) and NJ (Fig. 3), the group cluster of the trees have mix individuals of those two different species. This bring so many speculations and questions on the Epinephelus hexagonatus, are they belong in the same group to the species of Epinephelus fuscoguttatus? Or it happens because of the interference in their genetic makeup? Or probably had natural hybrid between those two species which cause it to carry some of the Epinephelus fuscoguttatus and Epinephelus hexagonatus genes? Or Epinephelus hexagonatus is misclassified as Epinephelus fuscoguttatus, which would therefore have the Epinephelus 
hexagonatus in the Epinephelus fuscoguttatus group and these has been reported by reference [24] that done a study on Barbus (Cyprinidae).

The answer maybe lies within the hatchery, ponds, or tanks that kept those fishes for broodstock management. Malaysia is striving for big commercialization of the aquaculture and fishery industry. However, there is no complete documentation [25] available on the broodstocks species and management. Most of the hatchery institutions bought their fry or fish larvae and the broodstock from local farms. Unfortunately, those farmers cannot identify any different species correctly just based on morphology itself and do not know how to handle the evolutionary aspects. As a consequence, the history, origin, size and sex ratio composition of the breeding stocks are frequently not optimally controlled. This bring so much concerned that repeated, uncritically advocacy has the potential to diminish recognition of the remaining gaps in thus knowledge and there, diminish support for continued research and development of an important management tool [26] raise expectations in the fishing community and in conservation circles that might prove unachievable; lead to neglect of other effective techniques for managing fishing effort; and result in expenditure of scarce financial and other resources in the creation and management of no-take reserves that are inappropriately sized or sited.

In an attempt to identify Epinephelus fuscoguttatus at the early stage of this study, its sequences were subjected to BLAST analysis with the available sequences in the Genbank. The results showed 98\% - 99\% similarity with Epinephelus hexagonatus. From the analysis, genetic distances were needed to be done to know exactly which group or species it belongs to.

\section{CONCLUSION}

Consequently, genetic monitoring of hybridization programs must be applied in a routine way, mainly to verify if trade and management of hybrids are correctly carried out in the fish breeding farm which can result in fertile hybrids and the variability of backcrossing. Furthermore, reports of these possible hybrids are not found, since Epinephelus hexagonatus have great morphologic similarity with the Epinephelus fuscoguttatus species, and genetic markers were not accessible in the identification of possible hybrids collected in the nature.

Therefore the correct identification could be very useful in developing broodstocks of Epinephelus fuscoguttatus which are known to be popular cuisines in Asia compared to the Epinephelus hexagonatus for profitable and sustainable aquaculture of this species. Moreover, correct identification of grouper species might support grouper conservation programs.

\section{ACKNOWLEDGMENT}

This work was supported by research grant number 0705-ABI-AB005 from the Agro-Biotechnology Institute, (ABI) under the Ministry of Science, Technology and Innovation (MOSTI), Malaysia.

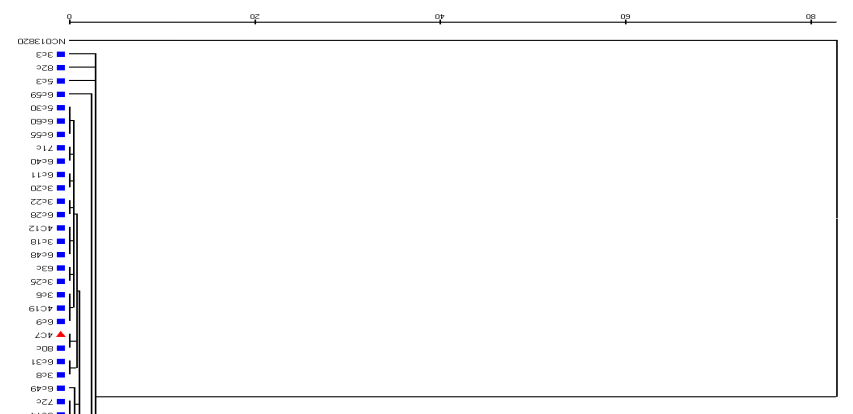

Figure 1. Evolutionary relationships of 160 taxa: blue represent Epinephelus fuscoguttatus and red represent Epinephelus hexagonatus.

The evolutionary history was inferred using the Maximum Parsimony method. The bootstrap consensus tree inferred from 1000 replicates is taken to represent the evolutionary history of the taxa. Branches corresponding to partitions reproduced in less than $60 \%$ bootstrap replicates are collapsed. The percentage of replicate trees in which the associated taxa clustered together in the bootstrap test (1000 replicates) are shown next to the branches. The MP tree was obtained using the Close-Neighbor-Interchange algorithm with search level 3 in which the initial trees were obtained with 
the random addition of sequences (100 replicates). The tree is drawn to scale, with branch lengths calculated using the average pathway method and are in the units of the number of changes over the whole sequence. All positions containing gaps and missing data were eliminated from the dataset (Complete Deletion option). There were a total of 340 positions in the final dataset, out of which 8 were parsimony informative. Phylogenetic analyses were conducted in MEGA 4.0.

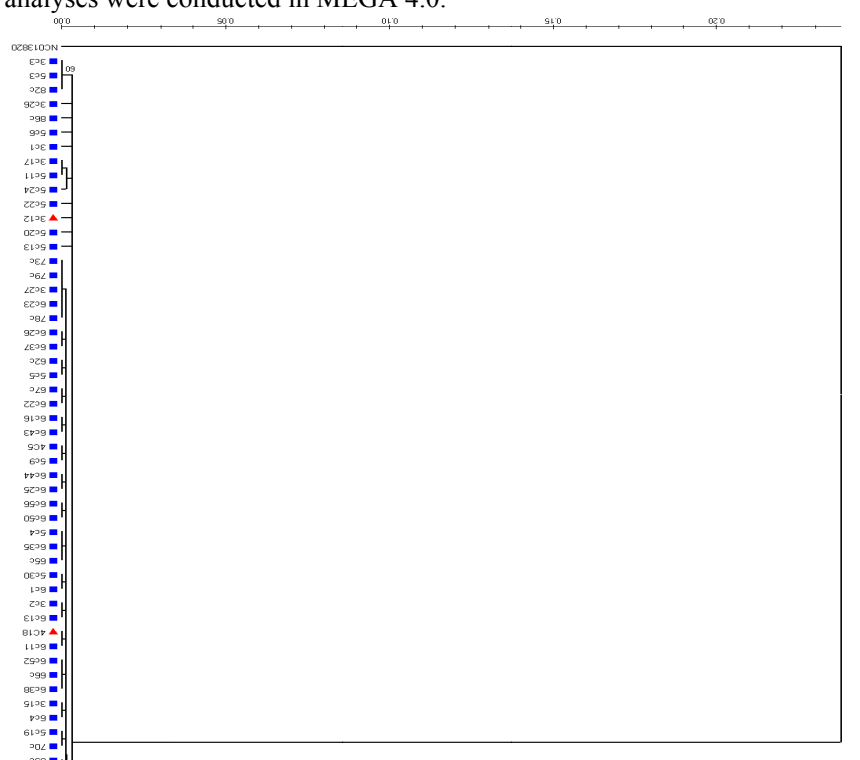

Figure 2. Evolutionary relationships of 160 taxa: blue represent Epinephelus fuscoguttatus and red represent Epinephelus hexagonatus.

The evolutionary history was inferred using the Minimum Evolution method. The bootstrap consensus tree inferred from 1000 replicates is taken to represent the evolutionary history of the taxa analyzed. Branches corresponding to partitions reproduced in less than $60 \%$ bootstrap replicates are collapsed. The percentage of replicate trees in which the associated taxa clustered together in the bootstrap test (1000 replicates) are shown next to the branches. The phylogenetic tree was linearized assuming equal evolutionary rates in all lineages. The tree is drawn to scale, with branch lengths in the same units as those of the evolutionary distances used to infer the phylogenetic tree. The ME tree was searched using the Close-NeighborInterchange (CNI) algorithm at a search level of 3. The Neighbor-joining algorithm was used to generate the initial tree. All positions containing gaps and missing data were eliminated from the dataset (Complete deletion option). There were a total of 340 positions in the final dataset. Phylogenetic analyses were conducted in MEGA 4.0.

\section{REFERENCES}

[1] Chiu, S.T., Tsai, R.T, Hsu, J.P., Liu, C.H., Cheng, W., 2008. Dietary sodium alginate administration to enhance the non-specific immune responses, and disease resistance of the juvenile grouper Epinephelus fuscoguttatus. Aquaculture., 277, 66-72.

[2] Yeh, S.L., Kuo, C.M., Ting, Y.Y., and Chang, C.F., 2003. Androgens stimulate sex change in protogynous grouper, Epinephelus coioides: spawning performance in sex-changed males. Comp. Biochem. Physiol. C., 135, 375-382.

[3] Ding, S., Xuan, Z., Feng, G., Jun, W., Yongquan, S., Qiyong, Z., and Qifu, L., 2006. Molecular phylogenetic relationships of China Seas groupers based on cytochrome b gene fragment sequences. Sci. China Ser. C-Life Sci., 49, 3.

[4] Craig, M.T., and Hastings, P.A., 2007. A molecular phylogeny of the groupers of the subfamily Epinephelinae (Serranidae) with a revised classification of the Epinephelini. Ichthyol. Res., 54, 1-17.

[5] Nguyen, T.T.T., Uthairat, N.N., Srijanya, S., Chen, Z.M., 2008. A study on phylogeny and biogeography of mahseer species (Pisces: Cyprinidae) using sequences of three mitochondrial DNA gene regions. Mol. Phylogenet. Evol., 48, 1223-1231.

[6] Merritt T.J.S., Shi. L., Chase, M.C., Rex, M.A., and Etter, R.J., 1998. Universal cytochrome b primers facilitate intraspecific studies in molluscan taxa. Mol Mar Biol Biotechnol., 7(1), 7-11.

[7] Zhu, Z.Y., and Yue, G.H., 2008. The complete mitochondrial genome of red grouper Plectropomus leopardus and its applications in identification of grouper species. Aquaculture., 276(1-4), 44-49.

[8] Craig, M.T., Pondella, D.J., Franck, J.P.C., and Hafner, J.C., 2001. On the Status of the Serranid Fish Genus Epinephelus: Evidence for Paraphyly Based upon 16S rDNA Sequence. Mol. Phylogenet. Evol., 19(1), 121-130.

[9] Palumbi, S.R. (1996). Nucleic acid II: the polymerase chain reaction. In: Hillis, D.M., Moritz, G., Mable, B.K. (eds.). Molecular Systematics. Sunderland, Mass.: Sinauer Associates, 205-247.

[10] Thompson J.D., Higgins D.G., Gibson T.J., 1994. CLUSTAL W: improving the sensitivity of progressive multiple sequence alignment through sequence weighting, position-specific gap penalties and weight matrix choice. Nucleic Acids Res., 22 (22); 4673-80.

[11] Tamura K, Dudley J, Nei M \& Kumar S (2007) MEGA4: Molecular Evolutionary Genetics Analysis (MEGA) software version 4.0. Molecular Biology and Evolution., 24,1596-1599.

[12] Felsenstein J., 1985. Confidence limits on phylogenies: An approach using the bootstrap. Evolution., 39:783-791.

[13] Nei M., and Kumar S., 2000. Molecular Evolution and Phylogenetics. Oxford University Press, New York.

[14] Saitou N., and Nei M., 1987. The neighbor-joining method: A new method for reconstructing phylogenetic trees. Molecular Biology and Evolution., 4,406-425.

[15] Ilves, K.L., and Taylor, E.B., 2008. Molecular resolution of the systematic of a problematic group of fishes (Teleostei: Osmeridae) and evidence for morphological homoplasy. Mol. Phylogenet. Evol., 50, 163-178.

[16] Ku, C.C., Teng, Y.C., Wang, C.S. and Lu, C.H., 2009. Establishment and characterization of three cell lines derived from the rockfish grouper Epinephelus quoyanus: Use for transgenic studies and cytotoxicity testing. Aquaculture., (in press). 
[17] Zhou, Z., Liu, Y., Shi, P., He, S., Yao, B., and Ring, E., 2009. Molecular characterization of the autochthonous microbiota in the gastrointestinal tract of adult yellow grouper (Epinephelus awoara) cultured in cages. Aquaculture., 286(3-4), 184-189.

[18] Ramon, M.L., and Knope, M.L., 2008. Molecular support for marine sculpin (Cottidae; Oligocottinae) diversification during the transition from the subtidal to intertidal habitat in the Northeastern Pacific Ocean. Mol. Phylogenet. Evol., 46, 475-483.

[19] Peng, Z., Shunping, H., and Yaoguang, Z., 2003. Phylogenetic relationships of glyptosternoid fishes (Siluformes: Sisoridae) inferred from mitochondrial cytochrome b gene sequences. Mol. Phylogenet. Evol., 31, 979-987.

[20] Czyzowski, P., Mirosław, K., and Leszek, D., 2008. Molecular investigation of phylogenetic relationships and phylogeography of polish pheasants using the mitochondrial cytochrome $b$ gene sequence. EJPAU., 11, 4.

[21] Keim P., Price L.B., Klevytska A.M., Smith K.L., Schupp J.M., Okinaka R., Jackson P.J., and Hugh-Jones M.E., 2000. MultipleLocus Variable-Number Tandem Repeat Analysis Reveals Genetic Relationships within Bacillus anthracis. Journal of Bacteriology, 182 (10); 2928-2936,

[22] Liu, Z.J., Cordes, J.F., 2004. DNA marker technologies and their applications in aquaculture genetics. Aquaculture., 238, 1-37.

[23] Slapeta J., Moreira D., and Lopez-Garcia P., 2005. The extent of protist diversity: insights from molecular ecology of freshwater eukaryotes Proc. R. Soc. B., 272; 2073-2081

[24] Markova S., Sanda R., Crivelli A., Shumka S., Wilson I.F., Vukic J., Berrebi P., Kotlík P., 2010. Nuclear and mitochondrial DNA sequence data reveal the evolutionary history of Barbus (Cyprinidae) in the ancient lake systems of the Balkans. Molecular Phylogenetics and Evolution., 55, 488-500

[25] Weeks P.J.D., O’Neill M.A., Gaston K.J., Gauld I.D. 1999. Speciesidentification of wasps using principal component associative memories. Image and Vision Computing., 17, 861-866.

[26] Piller K.R., and Bart Jr H.L.. 2009. Incomplete sampling, outgroups, and phylogenetic inaccuracy: A case study of the Greenside Darter complex (Percidae: Etheostoma blennioides). Molecular Phylogenetics and Evolution 53 (2009) 340-344.

Figure 3. Evolutionary relationships of 160 taxa: blue represent Epinephelus fuscoguttatus and red represent Epinephelus hexagonatus.

The evolutionary history was inferred using the Neighbor-Joining method. The optimal tree with the sum of branch length $=0.95095399$ is shown. The percentage of replicate trees in which the associated taxa clustered together in the bootstrap test (1000 replicates) are shown next to the branches. All positions containing gaps and missing data were eliminated from the dataset (Complete deletion option). There were a total of 372 positions in the final dataset. Phylogenetic analyses were conducted in MEGA 4.0.

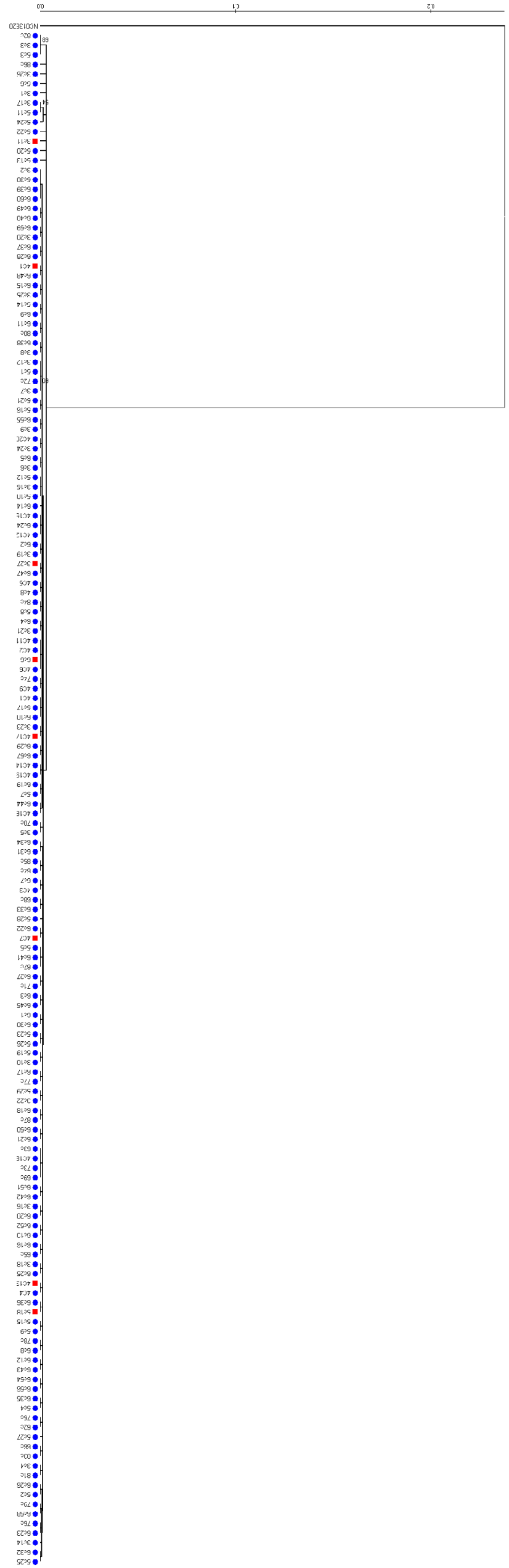

